\title{
NutriLive: An Integrated Nutritional Approach as a Sustainable Tool to Prevent Malnutrition in Older People and Promote Active and Healthy Ageing-The EIP-AHA Nutrition Action Group
}

\author{
Maddalena Illario, ${ }^{1,2}$ Angela Serena Maione, ${ }^{2}$ Maria Rosaria Rusciano, ${ }^{2}$ \\ Edwig Goossens, ${ }^{3}$ Amelia Rauter, ${ }^{4}$ Nidia Braz, ${ }^{5}$ Harriet Jager-Wittenaar, ${ }^{6}$ \\ Carolina Di Somma, ${ }_{1}^{1}$ Catherine Crola, ${ }^{2}$ Maria Soprano, ${ }^{2}$ Laura Vuolo, ${ }^{1}$ Pietro Campiglia, ${ }^{7}$ \\ Guido Iaccarino, ${ }^{8}$ Helen Griffiths, ${ }^{9}$ Tobias Hartman, ${ }^{10}$ Donatella Tramontano, ${ }^{11}$ \\ Annamaria Colao, ${ }^{12}$ and Regina Roller-Wirnsberger ${ }^{13}$
}

${ }^{1}$ Federico II University and Hospital, Campania EIP-AHA Reference Site, 80131 Naples, Italy

${ }^{2}$ Department of Translational Medical Science, Federico II University, 80131 Naples, Italy

${ }^{3}$ Center for Gastrology, 3000 Leuven, Belgium

${ }^{4}$ Faculty of Science, University of Lisbon, 1749-016 Lisbon, Portugal

${ }^{5}$ University of Algarve, 8005-139 Faro, Portugal

${ }^{6}$ Hanze University of Applied Sciences, 9747 AS Groningen, Netherlands

${ }^{7}$ Department of Pharmacy, University of Salerno, 84084 Fisciano, Italy

${ }^{8}$ Department of Medicine and Surgery, University of Salerno, 84084 Fisciano, Italy

${ }^{9}$ ARCHA, Aston University, Birmingham B47ET, UK

${ }^{10}$ The LipiDiDiet Consortium, Saarland University, 66123 Saarbrücken, Germany

${ }^{11}$ Department of Molecular Medicine and Medical Biotechnology, Federico II University, 80131 Naples, Italy

${ }^{12}$ Department of Clinical Medicine and Surgery, Federico II University, 80131 Naples, Italy

${ }^{13}$ Medical University of Graz, 8010 Graz, Austria

Correspondence should be addressed to Maddalena Illario; illario@unina.it and Regina Roller-Wirnsberger; regina.roller-wirnsberger@medunigraz.at

Received 30 June 2015; Revised 14 October 2015; Accepted 26 October 2015

Academic Editor: Panteleimon Giannakopoulos

Copyright (C) 2016 Maddalena Illario et al. This is an open access article distributed under the Creative Commons Attribution License, which permits unrestricted use, distribution, and reproduction in any medium, provided the original work is properly cited.

The present document describes a nutritional approach that is nested in the European Innovation Partnership for Active and Healthy Aging (EIP-AHA) and aims to provide the first common European program translating an integrated approach to nutritional frailty in terms of a multidimensional and transnational methodology. The document has been developed by the A3 Nutrition Action Area of the EIP-AHA and aims at providing a stepwise approach to malnutrition in older citizens, identifying adequate interventions based on a unified assessment and ICT-supported solutions. "NutriLive" is an integrated nutritional approach, represented by a structured Screening-Assessment-Monitoring-Action-Pyramid-Model (SAM-AP). Its core concept is the stratification of the nutritional needs, considered by the working group as the key for targeted, effective, and sustainable interventions. "NutriLive" tries to close gaps in epidemiological data within an aging population, creating a unified language to deal with the topic of nutrition and malnutrition in Europe. By assembling all the validated screening, assessment, and monitoring tools on malnutrition in a first pyramid, which is interrelated to a second intervention pyramid, the A3 Nutrition WG identifies a common, integrated vision on the nutritional approach to frailty, which applies to the various health care settings. 


\section{Introduction}

The European population is getting older but often not in good health and with reduced quality of life. Good nutrition and adequate physical activity have an important role to ensure that people not only can live longer but also can live healthy, active, and independent lives. In order to successfully increase healthy life years, it is essential to reduce and delay for as long as possible age-related functional decline and disability by targeting all their different determinants, among which nutrition plays a pivotal role. There is a case for tackling malnutrition at European level [1] due to high prevalence, numerous and costly consequences, and availability of relatively inexpensive interventions.

Differences in the study design of many clinical trials and observational studies often provided discordant findings and conclusions on malnutrition in older adults due to selection bias of participants especially in older adults and to smaller sample sizes of clinical trials for their strict inclusion and exclusion criteria. Of note, the life course approach to ageing has been only minimally addressed, although ageing is a lifelong process that begins in the womb, and many external influences, such as nutrition, influence it throughout life. Pregnancy and birth cohort studies might help elucidate these relationships, but very few existing birth cohorts follow into adulthood. The British birth cohort of the MRC National Survey of Health and Development (NSHD) began in 1946 and has been following participants up to age of 67 years. Birth cohorts such as that of NSHD are key to identifying key healthy ageing markers at different stages of life [2].

Older people are particularly vulnerable to malnutrition. Inadequate energy and nutrient balance and food intake are thought to be the major cause of weight loss/gain in older adults. Prescription of medicines, depression, and social isolation are contributors to inadequate energy and nutrient intakes in older adults as are the reduced sensations of taste and smell and poor dentition [3]. In addition, it has been suggested that a diminished ability to regulate food intake in older age is associated with the "anorexia of ageing" [4]. Roberts et al. have demonstrated that clear differences exist between young and old men in body weight change and voluntary food intake in response to overfeeding and underfeeding $[5,6]$. Food is the major source of bioactive compounds, crucial not only to prevent diseases but also to favor healthy aging. Adequate nutrient intake can reduce the risk of developing chronic pathologies including cardiovascular, neurodegenerative, and metabolic syndromes, decrease of bone density, muscle mass, and immune responsiveness [47]. A recent study by Viñas et al. [8] in 7 EU countries described that an inadequate intake of Vitamin B12 [9], Vitamin C, Vitamin D [10], folic acid [11], calcium, selenium, and iodine occurs in more than $20 \%$ of the older population (>64 years). Similar situations could be demonstrated for other regions such as US [12-14] and South America [15-17]. Deficits are more likely to occur in the homebound, frail older adult due to lack of mobility, difficulty to access healthy foods or grocery stores, and limited daily living activities, with difficulties in preparing meals themselves. Furthermore, ageing is recognized as independent risk factor for social isolation [18], which influences food intake. Migratory trends towards big cities leave countryside regions with changed demographic population patterns and limited access to local public transport and shopping possibilities. Trade dynamics have a potential negative impact on food supply, especially in disadvantaged groups, and trading policies are quite similar for all countries in the industrialized world. In many of those, food distribution is dominated by larger businesses, while smaller wholesalers and retailers are pushed out of the market. Local shops in poorer areas are often overpriced and low on choice and quality.

It has been demonstrated that older adults who are socially isolated also have an increased nutritional risk [19]. Older adults experiencing financial concerns such as poverty or low income may not be able to buy sufficient amount and quality of food. In Europe, financial aspects of access to healthy food become more and more prominent in the light of the current financial crises, especially in countries of lower economic status. In the US, it is common among elderly that choices need to be made between buying food and paying for other necessities such as medications, heat, rent, and others due to different public funding, social, and health care systems.

To achieve optimal health, it is important to consume a variety of foods from all food groups, which helps ensure the nutrients one needs. Dietary solutions and culinary approaches to nonillness related inadequate food intake represent an effective primary approach in many settings, in a flexible way and in different food cultures, at the same time setting the ground for cultural exchange and allowing each older person to have access to healthy, tasty, and nutritious meals. The consumption of functional foods like fortified drinks (milk, orange juice, and soy beverages) can also supply key nutrients as a secondary approach to provide the frail older adults with nutrients critical to their health [20-22]. Strategic policies in agriculture and fisheries may influence public health by affecting the supply, local availability, safety, affordability, and accessibility of foods. So far, agricultural policies have supported the production of sugar, fats and oils, meat and alcohol, while not equally sustaining the supply of fruit and vegetables [23]. Most of the food wheat is industrially processed, and more and more food is consumed outside the home, frequently with a higher content in energy and fat [24]. Inadequate nutrition worsens the age-related organ decline, thus contributing to the development of metabolic diseases, osteoporosis, sarcopenia, cognitive deficits [25], and anorexia [26], with a concurrent decline in the functionalities of the immune system [27]. The access to healthy food is crucial, particularly for frail older adults and even more for those who suffer from disabilities or illnesses that may affect their ability to function in everyday life. Preparing and eating a nutrient-dense diet, including providing simple, nutrientdense snacks that are easy to eat, can be important to improve the nutritional intake of older citizens across the globe.

Data within literature in the aging population in Europe [28-31] provide evidences about quantitative and qualitative malnutrition within this population, highlighting an increasing health, social, and economic impact [32]. Due to 
the fragmented and nation-centered nutrition policy within the European Union (EU) there seems to be a growing need for a common, integrated vision on the nutritional approach to active and healthy aging for the prevention of frailty and of functional decline.

The EU commission therefore provided one platform within the European Innovation Partnership on Active and Healthy Aging (EIP-AHA) for the development of a transnational think tank between stakeholders of all fields involved into food and nutrition supply across the continent. This multiprofessional group of representatives collaborates in terms of scientific projects as well as good practice models and their respective implementation and scaling-up in a common strategy. To settle grounds for a common understanding of the scientific and practical evidence for food supply in older European citizens the group elaborated a common vision for an integrated approach to nutrition. This approach is built on the primary hypothesis that prevalence of frailty and pre-frailty among older Europeans can be tackled by a multidimensional and transnational approach towards malnutrition among older adults. On this hypothesis, the group elaborated a new integrated concept of nutrition built on the Maslow Pyramid of individual needs $[33,34]$. This new model, called "NutriLive," builds the evidence for any implementation and scaling-up strategies developed by members of the large transnational European consortium working together in this field.

"NutriLive" takes into account domains such as social integration, psychosocial behavior, and cultural background of the aging population. This strategy covers personalized nutrition solutions with a strong public health focus. Nowadays, there is plenty of information on screening and assessment methods to test for nutritional deficits. However, only little evidence on nutritional interventions with varying endpoints of effectiveness is available.

Awareness on the aforementioned topics of stakeholders in politics and public health care planning as well as food production and supply is rather low. Built on the harsh grounds mentioned, the harmonization between scientific recommendations and approved health claims, including old and new functional products, is still missing and highly mandatory. The implementation of a common vision and strategy on the EU level will be evidence-based, for the availability of the assessment data, and effective as the latter will allow tailored interventions, in terms of maintaining health and functionality for older adults, as well as public healthcare planning, not only in Europe but also in all Western countries. Indeed, lack of data concerning the effectiveness, impact, and sustainability of targeted nutritional interventions that are based upon adequate assessment is pivotal to improve on a large scale overall health outcomes of EU citizens.

It was therefore the second hypothesis of this project that a common vision and strategy on the EU level will be highly specific and effective in terms of maintaining health and functionality for older adults in all EU countries. To answer this second hypothesis it became mandatory to build a transnational IT platform to collect individual as well as epidemiological data of older EU citizens and to follow these data in a longitudinal fashion.
The proposed model also takes into account contextual factors that influence healthy nutrition and as such supports adequate dietary regimens based upon the local availability of foods and is exportable to areas with different development levels.

\section{Strategies and Interventions for the "NutriLive" Approach}

2.1. The SAM-AP Model: Integrating Assessment with Interventions and Monitoring. "NutriLive" is a Pan-European approach nested in the European Innovation Partnership for Active and Healthy Aging (EIP-AHA). The partners work in all fields of nutrition, including academic institutions, health care providers, enterprises, patient groups, politicians, and many more. "NutriLive" reflects a common vision for nutritional approach of the European Union that may be transferred internationally. The strategy of the "NutriLive" concept is focused on a stepwise approach to malnutrition in the older citizens, which for the first time closes gaps of knowledge and imposes a common structured approach to stepwise adequate interventions (primary/secondary/tertiary), based on a unified assessment and modular ICT solutions. "NutriLive" takes advantage from transferring innovative tools to effective prevention, detection, and treatment measures. By assembling all the validated screening, assessment, and monitoring tools on malnutrition in an ICT-supported pyramid interrelated to the Action Pyramid, the "NutriLive" project group launches a common, integrated vision on the nutritional approach for prevention of functional decline and frailty across a whole continent taking into account the different geographical and sociocultural settings.

This goal will be achieved by the structured ScreeningAssessment \& Monitoring-Pyramid-Model (SAM-AP) into various health and social care systems (Figure 1). The Screening, Assessment \& Monitoring Pyramid shows the ranking of variables or tools needed to screen, assess, monitor, and treat malnutrition, and that eventually may result in consultation of a health care professional. The pyramid follows the idea of Maslow's hierarchy of needs $[33,34]$, reflecting the individualized approach to nutrition. As this concept works independently from geographical and health care settings (community level, hospitals, and nursing homes) it can be scaled up to any region worldwide. The SAM-AP Model is dynamic, meaning that the screening and assessment may result in food and nutritional care from any levels of the Action Pyramid on individual basis. By introducing the SAM-AP model, the view on nutrition broadens to the entire food chain and to its implications such as food safety and food supply. The culinary/clinical approach is similar to the partitioning of health care by WHO: primary, secondary, and tertiary. The dimensions reflect the culinary approach (primary food care), the culinary-clinical approach (collaboration primary food care/secondary nutritional care), and the clinical approach (secondary nutritional care) of the interventions. The SAMAP Model foresees stratified interventions that will therefore have impact on individual and public levels and builds the grounds for translating research evidence into care processes and public policy making across borders. 


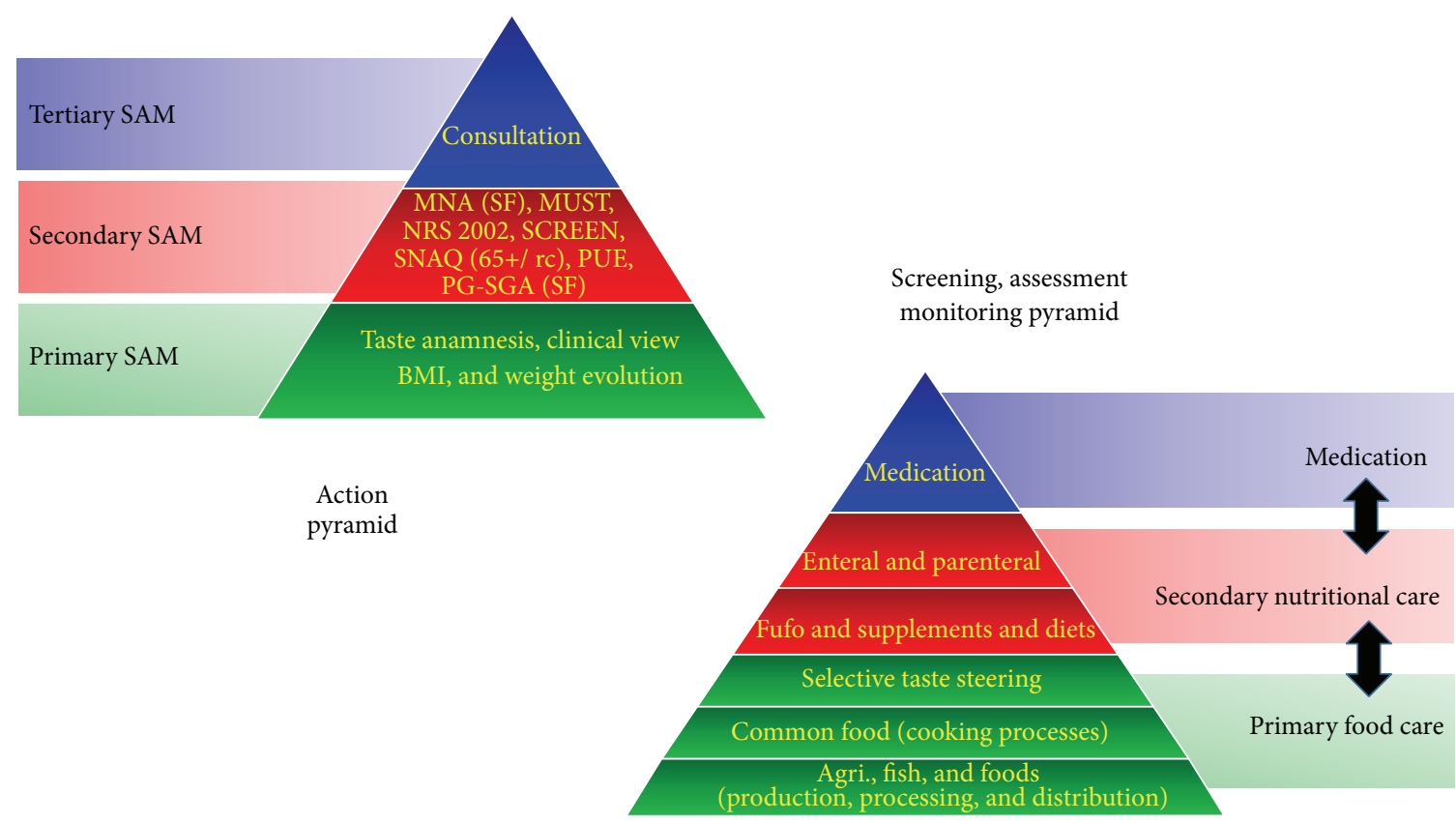

FIGURE 1: SAM-AP model: "NutriLive" approach represented as a modified food pyramid.

The SAM-AP model is based on a collaborative interventional model between the culinary approach and the clinical approach, which is person-centered, evidence-based, EPCinformed (evidence, practice, and creativity), and functionally interoperable with relevant topics, such as food safety, nutritional contents, and locoregional needs.

\subsection{Integration of the Strategies across the EU: The Nutritional} Platform of "NutriLive". Nutrition is largely influenced by availability of food, cultural traditions, social habits, and economic conditions. An effective and long lasting behavioral change can be achieved taking into account all contextual factors that characterize European countries and can contribute to enriching and fostering the cultural exchange using the nutritional driver.

Sharing effective assessment tools can be supported by adequate ICT solutions which can provide a common backbone to different tools in order to stratify nutritional needs across the EU to be matched with evidence-based and "geolocalized" primary nutritional interventions.

ICT has been proven to be an effective driver of societal innovation as long as its diffusion is paralleled by adequate literacy initiatives. ICT platforms can open online social media, create distributed knowledge, and support data collection from real environments [35]. Such an approach improves collective awareness about societal challenges and facilitates the identification of solutions that imply collaborative efforts, at the same time enabling new forms of social innovation, achieving changes in lifestyle, production, and consumption patterns, and setting up more participatory democratic processes.

ICT platforms help increase the awareness of the role that everyone can play to social issues, empowering and motivating citizens to make informed decisions as consumers, driving environmentally sustainable behavioural changes, stimulate participation, and remove barriers to an inclusive society.

An important feature of the Nutritional Platform will be its interoperability with other platforms that are being developed in the EU and are relevant for active and healthy ageing: PERSSILAA, Beyond Silos, DoReMi, NU-AGE, to name some, in order to potentiate the synergies between the different approaches to active and healthy ageing.

To transform the model of the SAM-AP into practice, Europe has established a technical service infrastructure for services and end users. The ICT support will provide an interoperable infrastructure to provide multiple types of services and facilitate person-generated data. Besides data management, this platform will support popular events, such as food blogger competitions on specific needs (diabetes, obesity, and celiac, cardiovascular disease), involving a scientific board, food bloggers, and chef's and hotel schools. Due to the flexibility of the technical settings, it will be possible to handle data coming from heterogeneous sources and to provide information to different end users. The platform will be available through a portal site allowing close collaboration between all actors involved, cross borders, at the lowest operational cost by scaling it up to the European digital level. The portal site is the entrance to various tools which are offered by the platform via responsive web site, meaning that the portal site can be used on a broad range of devices, from desktop computers or laptops to smartphones and tablets. The only condition is an internet connection, because the data is managed centrally and can be consulted from everywhere.

The platform is interoperable with the various preexisting services and information systems. This setting will enable longitudinal monitoring of individual histories and interventions, will open space for public communication and 
knowledge provision in terms of blog's recipes, and therefore will close intercultural and interregional gaps. The "NutriLive" platform will also give chance to get an overall regional picture for cross-sectional analysis, risk profiling, and regional changes of nutritional patterns. The platform follows latest IT safety and control standards.

\subsection{Screening and Assessment of Malnutrition as a Risk Factor} for Active and Healthy Aging: Focusing on Older Adults. Individual as well as public awareness for the topic of malnutrition is still low. Despite convincing research data towards an effectiveness of screening for malnutrition and its risk factors (e.g., loss of appetite, chewing [36] and swallowing problems, depression, and polypharmacy therapy [37]), risk assessment and evidence for the best fitting nutritional interventions in terms of a broad public health approach on EU level are still missing. To facilitate the evaluation of the effectiveness of screening, assessment, and interventions, the process as a whole should be integrated across all health care settings. The generation of data deriving from the different possible scenarios will provide as broad a sample for $\mathrm{EU}$ representative locations as possible within the partners of the EIP-AHA. In the context of a life course approach to active and healthy ageing, if we focus on the population of older adults, the following different subsets are recommended for the evaluation of needs of the different target populations: older adults $>65$ years in the general population (including home care), in assisted living facilities, at the primary care level and inpatients at hospitals.

To improve the awareness on the role of nutrition in health and disease among the older adults, a person-centric and health/ICT literacy oriented interventional approach is recommended. Older citizens need to be empowered to perform self-screening and self-assessment, and in our model they are actively involved in the choice of the interventions. Owning personal nutrition track records across all care settings will improve individual awareness for deficits [3842] and preferences and will link a preventive public health approach with all settings, as nutrition is part of successful living and well-being. Broad campaigns on EU level will support self-empowerment towards healthy nutrition on the primary care level.

In this nutritional care process, a stratification of the older citizens is necessary, based upon their needs. A set of advanced, innovative biomarkers and information from big data (metabolomics, microbiomics, proteomics, genomics, and drug consumption) may support a more personalized approach and potentially improve the overall outcome of the interventions [39, 40,43]. Different sets of biomarkers will support early interventions focused to respond to different nutritional needs: lack of specific nutrients in the context of multimorbidity (e.g., osteoporosis and Vitamin D deficiency in subjects with dyslipidemia and chronic renal failure) and of cardiovascular risk, metabolic disorders, and many others. The identification of innovative biomarkers and parameters especially concerning inflammatory profile, lipid, and glucose metabolism could improve the identification of population subsets and risk profiles that might take advantage of focused nutritional interventions and predict/monitor the therapeutic response of individuals affected by nutritionally regulated diseases.

In order to provide a holistic approach when designing the interventions we need to take into account the food intake and the social context.

\subsection{Knowledge Generation on Individual Multidirectional} Clustering of Citizens and Food. Within "NutriLive," a needsbased stratification of older citizens is mandatory to allow structured and individualized nutritional care process planning as well as public planning. Knowledge generation on the impact of lifestyle, socioeconomic settings, and cultural, ethnical, migratory, and gender aspects as well as functional and disease patterns on nutritional habits at the epidemiological level of a whole continent are still lacking. Thus, our second hypothesis will be fulfilled by the "NutriLive" platform, which will allow the analysis of aggregated data and subsequent risk clustering in order to improve recruitment and outcome parameters for planning nutritional interventions and trials and will therefore close the gap on best evidence research practice for nutritional interventions.

This clustering will integrate sets of advanced, innovative biomarkers, information from big data (metabolomics, microbiomics, proteomics, and genomics), and assessment tools to allow a personalized, early approach, improving the overall outcome of the interventions [39, 40,43]. Particularly in the light of increasing numbers of overweight citizens with concomitant muscle wasting, the identification of innovative biomarkers and parameters, on inflammatory profile, lipid, and glucose metabolism, is mandatory to improve early identification of population subsets that might take advantage of focused nutritional and lifestyle interventions of nutritionally regulated diseases (obesity and type 2 diabetes). Looking at epidemiological data from US and South America, this aspect seems to be intrinsically interesting for other parts of the world as well.

The appropriate use of dietary supplements and of fortified foods, together with the development of innovative value-added products incorporating bioactive components (antioxidants, pigments, polysaccharides, proteins, peptides, hydrolyzed proteins, amino acids, fatty acids, and lipids), represents an excellent secondary approach to improving the nutrition of older citizens and their overall health outcomes. More in-depth in vitro and in vivo studies are needed though in order to exploit all the bioactive compounds available from biological resources, using state-of-the-art technologies, aimed at elucidating and understanding the cell and molecular mechanisms of action of these bioactive compounds and their implications in human physiology. The study of the many gastrointestinal effects of different bioactive compounds administration in the older citizens as well as their effects to the microbiota composition and to the intestinal immune response is relevant and will be matched by the development of controlled delivery systems (micro- and nanoparticles of biodegradable and biocompatible polymers) for the identified bioactive compounds. The development of active packaging applications utilizing identified bioactive ingredients and the assessment of the biological and chemical 
stability of food products containing bioactive ingredients following common food manufacturing processes and of bioaccessibility of the bioactive ingredients (i.e., the degree of bioactivity available for absorption in the gut after digestion) will contribute to enriching the nutritional approach to preventing functional decline and frailty during the advanced life course.

2.5. Culinary Approach. The culinary approach is an effective primary intervention strategy, where the collaboration of chefs with dietitians and nutritionists can provide education on problem solving about specific topics, like swallowing problems, food quality indicators, and cooking techniques. The challenge is that most cooking skills mastered by ancient housewives and cooks got lost during industrial revolution and, at present, most people lack the basic information to make the best food and culinary choices. When old age functional decline makes it harder to shop, cook, chew, swallow, digest, or metabolize, the clinical picture is loss of functional capacity and subsequent malnutrition. Early prevention by adaptions in primary food supply would enhance the chance to choose differently, "wisely," and sustainably. Older consumers very often refuse caterers and health facilities food services because their products lack cultural heritage and taste quality, despite providing the right choice of nutrients. The use of adequate ICT tools will support our proposed primary approach, through dedicated systems for knowledge monitoring and management, implemented in our "NutriLive" low entry Digital Food Platform. For example, the platform offers collecting multilingual food recipes and menus that represent a relevant part of the cultural heritage of the European countries: indeed, some eating habits develop over many years and are handed down in the oral tradition from one generation to the next. Collecting recipes, menus, and the related tales and stories of our tradition would represent an added value and would support cultural exchange, which becomes more and more challenging due to increased migration trends in Europe and in the US.

2.6. Food Supply, Agriculture, and Nutritional Interventions. Older citizens who are losing functionality and social integration often have to rely on public food supply. In agreement with WHO European Action Plan for Food and Nutrition Policy 2007-2012 [44], the outlined activities are aimed at improving the nutritional quality of food supply and food safety, at promoting appropriate micronutrient fortification of staple food items, and at developing complementary foods with adequate micronutrient content.

In the "NutriLive" concept recommendations of WHO are incorporated. As indicated in our second hypothesis, the activities will be addressed to close the gaps between food production, delivery, and nutritional interventions on the micro level, such as the characterization (chemical and biological) of foods from different regional dietary habits and practices and their comparison to highlight similarities and differences. Therefore, identifying the chemical composition of a large variety of nutrients will contribute to meeting the specific needs of the different group of the aging population, stratified and clustered according to geographical area and major pathologies. This will give the possibility to introduce new food products in accordance with healthy eating policy (production of tasty foods enriched with bioactive nutritional derived chemicals, presented in a consumer acceptable form) for older people. A major goal is the development of prototype ingredient products for subsequent inclusion in food matrices, animal-feeding trials, and pilot scale dietary intervention studies. The availability of a platform supporting integrated assessment and data storage will also allow monitoring along the different dimensions in a personalized way. Discretional access to specific datasets will be possible depending upon user's profile (health care professional, citizen, chef, and others), ensuring correct use of aggregated data and trends.

\section{Discussion and Concluding Remarks}

This paper describes the "NutriLive" project of the EIP-AHA, which is the first Pan-European attempt to foster social inclusion of older citizens by maintaining individual and overall functional capacity keeping up the right for adequate nutrition across the entire lifespan. The project was built on the primary hypothesis that prevalence of frailty and prefrailty among older Europeans can be tackled by a multidimensional and transnational approach towards malnutrition among older adults. On this hypothesis, the group elaborated a new integrated concept of nutrition based on the Maslow Pyramid of individual needs. Using a newly developed Nutrition ICT platform to collect data (Figure 2), the group will build the evidence for any implementation and scaling-up strategies on nutrition and food supply in older adults in Europe.

"NutriLive" closes the current gaps in epidemiological data within an aging European population, creating a unified language to deal with the topic of nutrition and malnutrition in Europe. "NutriLive" chooses a broad and multidimensional approach to translate personalized interventions into scaling-up to public health level, therefore contributing to the EC goal to increase the average healthy lifespan in the EU by two years by 2020 in a more accessible and sustainable way. "NutriLive" takes advantage of the knowledge and service modules for screening, monitoring, and end user support for lifestyle that were generated by European projects (e.g., LipiDiDiet, PERSSILAA, Nutrition Day in Hospital and Nursing Homes in Europe, Nutritional Screening for Hospitalized Patients Graz, Goal, SHARA, DoReMi, NU-AGE, and Beyond Silos) and closes existing gaps generating a common European platform based upon a common vision. The main goal of the current strategy builds on support of actions carried out by the partners of the A3 Nutrition subgroup and to all other interested stakeholders (interconnected and complementary) to provide ICT-supported personalized nutritional interventions to older European citizens aged >65 years. The interventions range from a culinary approach to dietary supplements and appropriate drugs, as well as assessment of potential interactions between food and drug, depending upon assessment of nutritional condition, to be carried out at different, critical stages of life, when inadequate nutritional intake can influence healthy aging. "NutriLive" follows the concept identified by the "PERSSILAA" project, in that it 


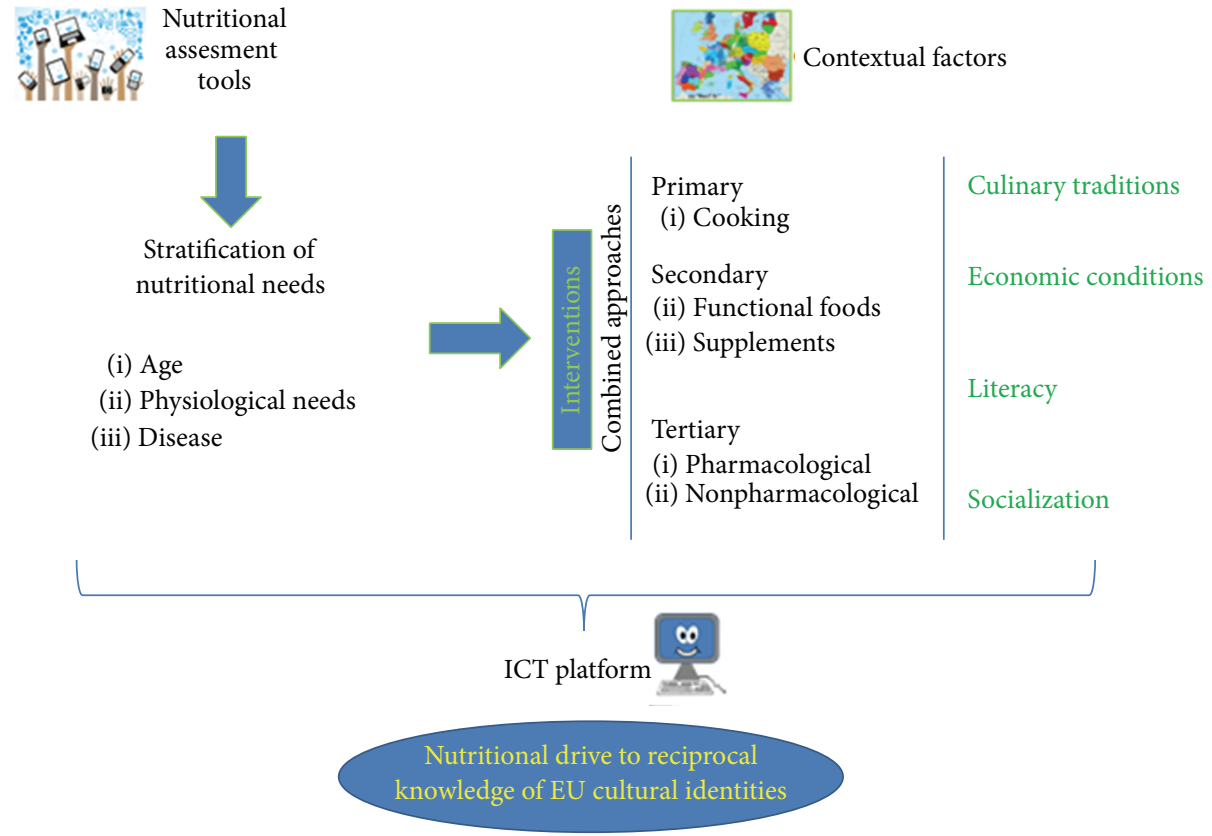

FIgUre 2: The concept of "NutriLive" ICT architecture.

innovates the way our services are intended and organized, from fragmented reactive disease management into preventive personalized services, which approach nutrition as a whole and appropriately target nutrition throughout the entire life course. The partners include policy makers, health care and social services, clinicians, pharmacists, informal caregivers, chefs, dietitians, scientists, and enterprises that will provide the assessment tools for personalized interventions sets designed for specific phases of the nutritional state. These stakeholders have been collaborating in the context of the EIP on AHA Nutrition Action Group since 2012 and elaborated a common work where each partner contributes to a specific deliverable. Periodic monitoring is ensured within the group by its flexible and horizontal government structure, which could take advantage of sharing knowledge, tools, and results through the platform.

Indeed, nutrition is intertwined with many variables that require different levels and intensity of interventions care and cure. The foreseen activities will also contribute to building business models for sustainable implementation and developing recommendations for European guidelines. The group provides a unique mix of skills, merging social, medical, and technological sciences with industry, academia, and end user organizations, taking advantage from ICT support to store validated screening tools, and carrying out data analysis, at the same time ensuring interoperability with existing platforms and internationally oriented modular training programs, which will take advantage from online training modules. By setting up an international platform, we can keep the operational cost as low as possible and link it to other existing data records. The community-based approach of the platform is limited not only to its usage but also to its development strategy. This means that further development of the platform will be a joint effort with other partners at local, national, and international levels.

\section{Future Perspectives of "NutriLive"}

The success of multi- and interdisciplinary interactions is pivotal to ensure effective strategies to tackle adequate nutrition and achieve active and healthy ageing. The user-friendly and multimodular platform, "NutriLive," will support large scale nutritional and lifestyle assessment and interventions, at the same time increasing our awareness about cost-effectiveness of the interventions, which will be assessed by adequate teams dedicated to the underpinning data gathering process. The integrated activities of accountants, managers, clinicians, and researchers in the different relevant fields will allow the identification of the cost drivers of the innovative interventions to support sustainable planning in the short, medium, and long term for social and health systems.

\section{Conflict of Interests}

All authors declare that there is no conflict of interests.

\section{Acknowledgments}

The authors thank Nunzia Montuori, DISMET, Federico II University, Naples, Italy, and Cornel C. Sieber, Institute for Biomedicine of Ageing (IBA), Nuremberg, Germany, for critical reading, the Policy Officers of the European Commission DG SANTE, Jorge Pinto-Antunes, Inés GarcíaSánchez, and Anna Carta, for managing and supporting the EIP AHA, all partners of the EIP on AHA A3 Action Group Nutrition Action Area who are involved to different extent in 
the common work, the Reference Site Collaborative Network of the EIP on AHA for providing their AG with their broader "system" perspective, the management and administration of Federico II University Hospital for ensuring support to the coordination of the EIP on AHA Nutrition Action Area, and Campania EIP on AHA Reference Site team.

\section{References}

[1] World Health Organization, Global Age Friendly Cities: A Guide, World Health Organization, Geneva, Switzerland, 2007.

[2] M. Wadsworth, D. Kuh, M. Richards, and R. Hardy, "Cohort profile: the 1946 national birth cohort (MRC National Survey of Health and Development)," International Journal of Epidemiology, vol. 35, no. 1, pp. 49-54, 2006.

[3] B. J. Stewart-Knox, E. E. A. Simpson, H. Parr et al., "Zinc status and taste acuity in older Europeans: the ZENITH study," European Journal of Clinical Nutrition, vol. 59, supplement 2, pp. S31S36, 2005.

[4] J. E. Morley, "Anorexia of aging: physiologic and pathologic," The American Journal of Clinical Nutrition, vol. 66, no. 4, pp. 760-763, 1997.

[5] S. B. Roberts, P. Fuss, M. B. Heyman et al., "Control of food intake in older men," The Journal of the American Medical Association, vol. 272, no. 20, pp. 1601-1606, 1994.

[6] S. B. Roberts, "Regulation of energy intake in relation to metabolic state and nutritional status," European Journal of Clinical Nutrition, vol. 54, supplement 3, pp. S64-S69, 2000.

[7] A. C. Milne, J. Potter, A. Vivanti, and A. Avenell, "Protein and energy supplementation in elderly people at risk from malnutrition," Cochrane Database of Systematic Reviews, no. 2, Article ID CD003288, 2009.

[8] B. R. Viñas, L. R. Barba, J. Ngo et al., "Projected prevalence of inadequate nutrient intakes in Europe," Annals of Nutrition and Metabolism, vol. 59, no. 2-4, pp. 84-95, 2011.

[9] NIH-Office of Dietary Supplements: Dietary Supplement Fact Sheet: Vitamin B12, 2011, https://ods.od.nih.gov/factsheets/VitaminB12-HealthProfessional/.

[10] G. Bjelakovic, L. L. Gluud, D. Nikolova et al., "Vitamin D supplementation for prevention of mortality in adults," The Cochrane Database of Systematic Reviews, no. 1, Article ID CD007470, 2014.

[11] NIH-Office of Dietary Supplements: Dietary Supplement Fact Sheet: Folate, 2012, http://ods.od.nih.gov/factsheets/FolateHealthProfessional/.

[12] A. A. Ginde, M. C. Liu, and C. A. Camargo Jr., "Demographic differences and trends of vitamin D insufficiency in the US population, 1988-2004," Archives of Internal Medicine, vol. 169, no. 6, pp. 626-632, 2009.

[13] Centers for Disease Control and Prevention, Second National Report on Biochemical Indicators of Diet and Nutrition in the U.S. Population, Centers for Disease Control and Prevention, DeKalb County, Ga, USA, 2012.

[14] T. C. Wallace, M. McBurney, and V. L. Fulgoni III, "Multivita$\mathrm{min} /$ mineral supplement contribution to micronutrient intakes in the United States, 2007-2010," Journal of the American College of Nutrition, vol. 33, no. 2, pp. 94-102, 2014.

[15] H. Sanchez, C. Albala, L. Lera, A. D. Dangour, and R. Uauy, "Effectiveness of the national program of complementary feeding for older adults in chile on vitamin B12 status in older adults; secondary outcome analysis from the CENEX Study
(ISRCTN48153354)," Nutrition Journal, vol. 12, no. 1, article 124, 2013.

[16] A. C. L. Cirino, R. de Vargas Zanini, and D. P. Gigante, "Consumption of foods with voluntary fortification of micronutrients in southern Brazil: prevalence and associated factors," Public Health Nutrition, vol. 17, no. 7, pp. 1555-1564, 2014.

[17] L. A. Martini, E. Verly Jr., D. M. L. Marchioni, and R. M. Fisberg, "Prevalence and correlates of calcium and vitamin D status adequacy in adolescents, adults, and elderly from the Health Survey-São Paulo," Nutrition, vol. 29, no. 6, pp. 845-850, 2013.

[18] S. Iliffe, K. Kharicha, D. Harari, C. Swift, G. Gillmann, and A. E. Stuck, "Health risk appraisal in older people 2: the implications for clinicians and commissioners of social isolation risk in older people," British Journal of General Practice, vol. 57, no. 537, pp. 277-282, 2007.

[19] J. L. Locher, C. S. Ritchie, D. L. Roth, P. S. Baker, E. V. Bodner, and R. M. Allman, "Social isolation, support, and capital and nutritional risk in an older sample: ethnic and gender differences," Social Science and Medicine, vol. 60, no. 4, pp. 747-761, 2005.

[20] W. Wouters-Wesseling, L. W. Wagenaar, M. Rozendaal et al., "Effect of an enriched drink on cognitive function in frail elderly persons," Journals of Gerontology A: Biological Sciences and Medical Sciences, vol. 60, no. 2, pp. 265-270, 2005.

[21] W. Wouters-Wesseling, A. P. Vos, M. Van Hal, L. C. P. G. M. De Groot, W. A. Van Staveren, and J. G. Bindels, "The effect of supplementation with an enriched drink on indices of immune function in frail elderly," Journal of Nutrition, Health and Aging, vol. 9, no. 4, pp. 281-286, 2005.

[22] W. Wouters-Wesseling, L. W. Wagenaar, L. C. P. G. M. de Groot, J. G. Bindels, and W. A. van Staveren, "Biochemical antioxidant levels respond to supplementation with an enriched drink in frail elderly people," Journal of the American College of Nutrition, vol. 22, no. 3, pp. 232-238, 2003.

[23] D. Stewart, A. Kennedy, and A. Pavel, "Beyond nutrition and agriculture policy: collaborating for a food policy," British Journal of Nutrition, vol. 112, supplement 2, pp. S65-S74, 2014.

[24] W. B. Traill, M. Mazzocchi, B. Shankar, and D. Hallam, "Importance of government policies and other influences in transforming global diets," Nutrition Reviews, vol. 72, no. 9, pp. 591-604, 2014.

[25] E. Sydenham, A. D. Dangour, and W.-S. Lim, "Omega 3 fatty acid for the prevention of cognitive decline and dementia," Cochrane Database of Systematic Reviews, vol. 6, Article ID CD005379, 2012.

[26] C. Moss, W. S. Dhillo, G. Frost, and M. Hickson, "Gastrointestinal hormones: the regulation of appetite and the anorexia of ageing," Journal of Human Nutrition and Dietetics, vol. 25, no. 1, pp. 3-15, 2012.

[27] K. Chandra R, "Nutrition and the immune system: an introduction," The American Journal of Clinical Nutrition, vol. 66, no. 2, pp. 460S-463S, 1997.

[28] Nutrition Day, http://www.nutritionday.org/.

[29] M. Pirlich and H. Lochs, "Nutrition in the elderly," Best Practice \& Research Clinical Gastroenterology, vol. 15, no. 6, pp. 869-884, 2001.

[30] R. Wirth, M. Streicher, C. Smoliner et al., “The impact of weight loss and low BMI on mortality of nursing home residentsresults from the nutritionDay in nursing homes," Clinical Nutrition, 2015. 
[31] The Lancet Nutrition Series report (JRC Science and Policy Reports), theRole of Nutrition in Active and Healthy Ageing, 2014.

[32] BAPEN, Redditch, UK, http://www.bapen.org.uk/.

[33] F. J. van Lenthe, T. Jansen, and C. B. Kamphuis, "Understanding socio-economic inequalities in food choice behaviour: can Maslow's pyramid help?" British Journal of Nutrition, vol. 113, no. 7, pp. 1139-1147, 2015.

[34] A. Maslow, "A theory of human motivation," Psychological Review, vol. 50, no. 4, pp. 370-396, 1943.

[35] Digital Agenda for Europe, "Collective Awareness Platforms for Sustainability and Social Innovation," https://ec.europa.eu/digital-agenda/en/collectiveawareness\#Article.

[36] I. Nykänen, E. Lönnroos, H. Kautiainen, R. Sulkava, and S. Hartikainen, "Nutritional screening in a population-based cohort of community-dwelling older people," European Journal of Public Health, vol. 23, no. 3, pp. 405-409, 2013.

[37] R. A. Heuberger and K. Caudell, "Polypharmacy and nutritional status in older adults: a cross-sectional study," Drugs and Aging, vol. 28, no. 4, pp. 315-323, 2011.

[38] T. Vikstedt, M. H. Suominen, A. Joki, S. Muurinen, H. Soini, and K. H. Pitkälä, "Nutritional status, energy, protein, and micronutrient intake of older service house residents," Journal of the American Medical Directors Association, vol. 12, no. 4, pp. 302307, 2011.

[39] L. M. Donini, C. Savina, and C. Cannella, "Eating habits and appetite control in the elderly: the anorexia of aging," International Psychogeriatrics, vol. 15, no. 1, pp. 73-87, 2003.

[40] J. E. Morley, "Weight loss in older persons: new therapeutic approaches," Current Pharmaceutical Design, vol. 13, no. 35, pp. 3637-3647, 2007.

[41] R. Visvanathan and I. M. Chapman, "Preventing sarcopaenia in older people," Maturitas, vol. 66, no. 4, pp. 383-388, 2010.

[42] A. H. Maslow, Motivation and Personality, Harper, New York, NY, USA, 1954.

[43] J. M. Boyce and G. R. Shone, "Effects of ageing on smell and taste," Postgraduate Medical Journal, vol. 82, no. 966, pp. 239241, 2006.

[44] World Health Organization, WHO European Action Plan for Food and Nutrition Policy 2007-2012, WHO, Geneva, Switzerland, 2008. 


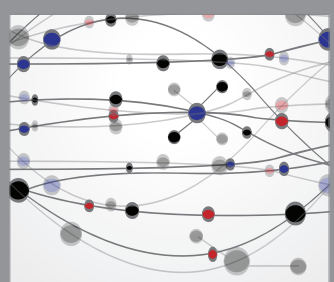

The Scientific World Journal
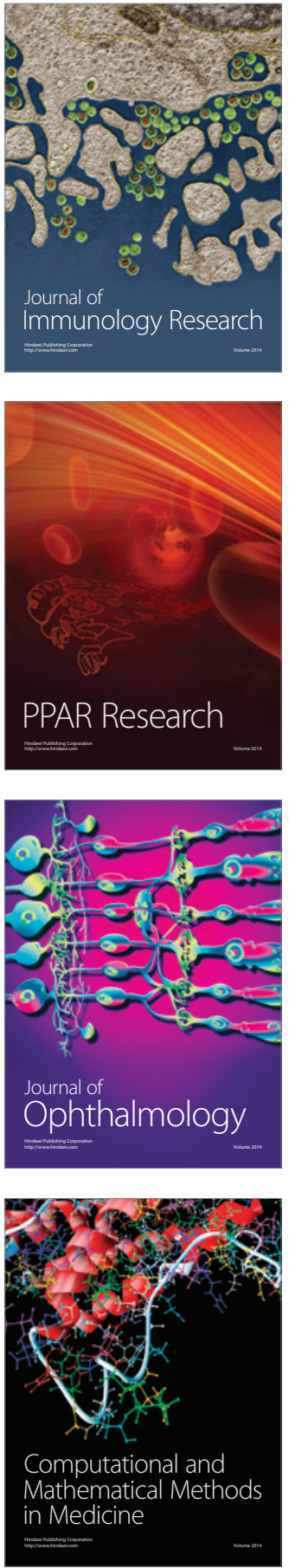

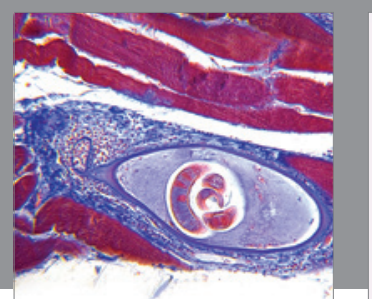

Gastroenterology Research and Practice

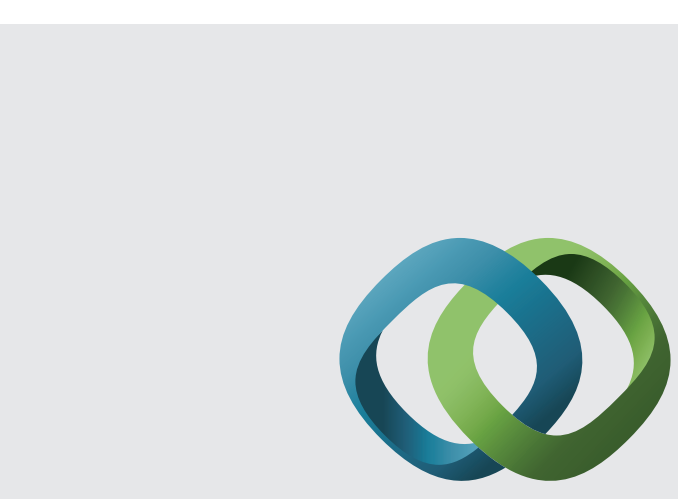

\section{Hindawi}

Submit your manuscripts at

http://www.hindawi.com
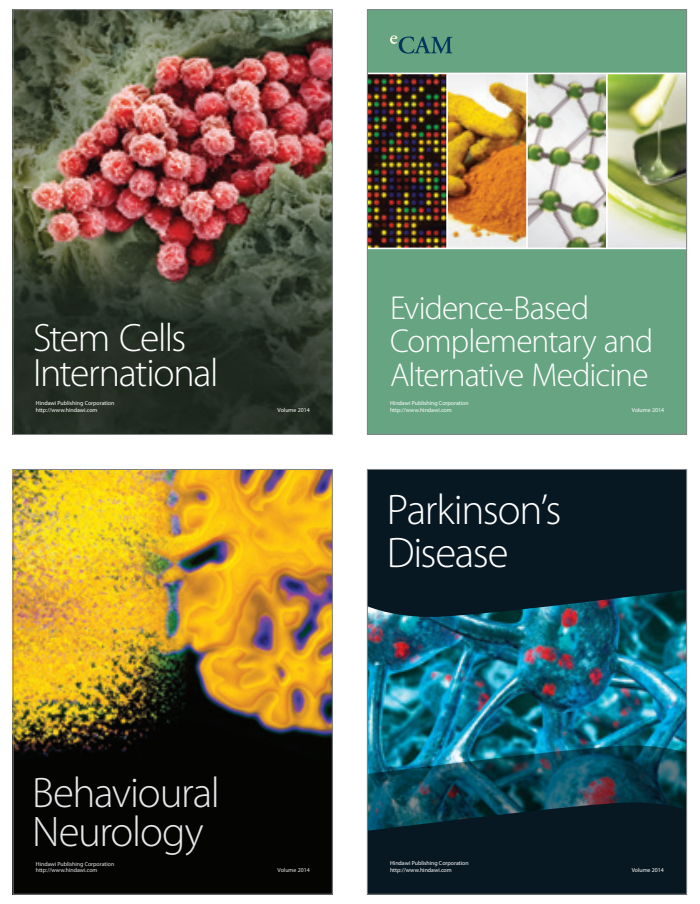
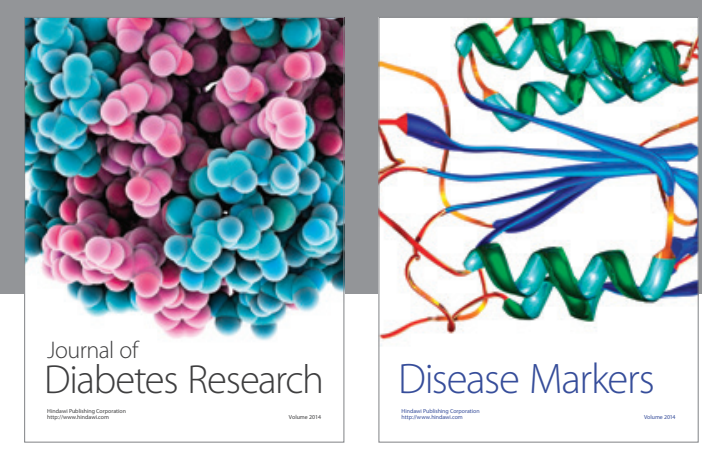

Disease Markers
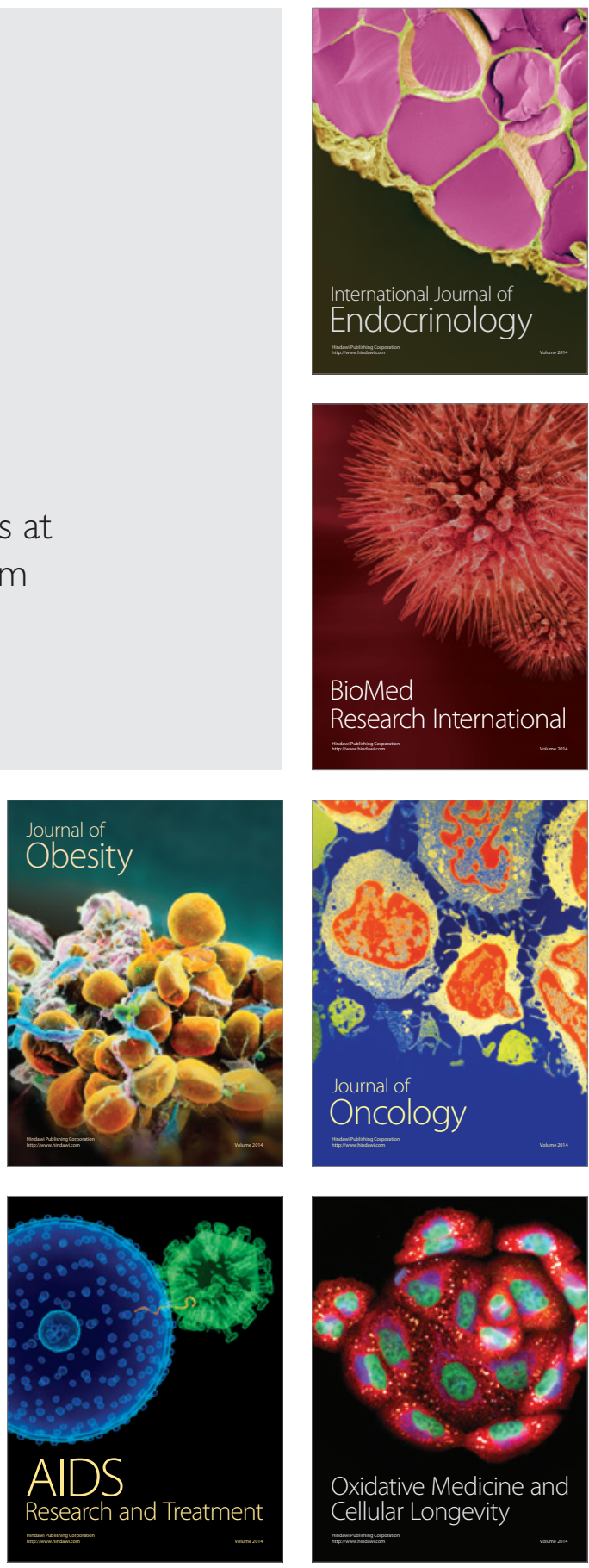